\title{
Welcome to the year 2015
}

\author{
Nadia Magnenat-Thalmann
}

Published online: 12 December 2014

(C) Springer-Verlag Berlin Heidelberg 2014

Welcome to 2015. Our journal has improved its impact factor, which is now 1.073. Thank you to all of you for your continuous collaboration and tremendous work.

Throughout last year, some members left the Editorial Board. We take this opportunity to thank the following members for their contribution to The Visual Computer:

- Tat-Seng Chua, National University of Singapore

- Ingemar J. Cox, University College London, UK

- Jan Kautz, University College London, UK

- Qunsheng Peng, Zhejiang University, China

- Leslie Piegl, University of South Florida, USA

- Jue Wang, Adobe, Seattle USA

We would also like to welcome the new Associate Editors who have joined the editorial board this year:

- Nadia Berthouze, University College London, UK

- Marina Gavrilova, University of Calgary, Canada,

- Hua Gang, Stevens Institute of Technology, USA

- Hua Huang, Beijing Institute of Technology, China

- Walter Kropatsch, Vienna University of Technology, Austria,

- Chau Lap-Pui, Nanyang Technological University, Singapore

- Guillaume Lavoue, LIRIS, INSA-CNRS, Lyon, France

- Jia Li, Yahoo Labs, USA

- Jakob Munkberg, Intel Corporation, Sweden
- Daniele Panozzo, ETH Zurich, Switzerland

- George Papagiannakis, University of Crete, Greece,

- Bodo Rosenhahn, Leibniz Universität Hannover, Germany

- Emily Whiting, Dartmouth College, USA

This year, 81 research papers were accepted for publication in the Visual Computer. Thank you very much to our 392 reviewers and to our 70 associate editors, that makes possible the selection of these high quality papers.

For the coming year, as usual, we schedule regular issues and a main special issue that will contain the 35 best papers from the Computer Graphics International Conference (CGI'2015), that will be held in Strasbourg, France. Next year, we will also have a special on Geometric Modelling and Learning for Visual Data Analysis. It is scheduled to appear by the end of 2015 .

We are grateful to Shi-Min Hu, Associate Editor-in-Chief, for his constant collaboration, as well as to the Editorial Assistants, Aryel Beck, Zhang Zhijun, and Yong-Jin Liu for their help and support.

We would also like to thank our Editorial Director at Springer, Beverley Ford, and Rachel Roberts, the Editor of the journal for their support. Thank you also to Beate Uhl, the Production Editor, and to Sneha Rahul for their tremendous work and constant assistance.

Nadia Magnenat Thalmann

Editor-in-Chief

N. Magnenat-Thalmann ( $\varangle)$

MIRALab-CUI, University of Geneva, Battelle, Building A,

7, Route de Drize, 1227 Carouge, Geneva, Switzerland

e-mail: thalmann@miralab.ch 


\section{Reviewers list}

Abhijeet Ghosh

Adolfo Munoz

Ahmet Akyuz

Aidong Lu

aleksandrs ecins

Alessandro Artusi

Alexander Bogomjakov

Anderson Maciel

Andrea Cerri

Andreas Tarnowsky

Annalisa Franco

Anshuman Razdan

Ashnil Kumar

Belen Masia

Ben Jones

Bernard Ghanem

Bernardt Duvenhage

Bernhard Preim

Bilal Alsallakh

Bin Fan

Bin Sheng

Bo Yang

Boleh Zhou

Bongjin Koo

Brandon Miles

Brian Allen

Byung Cheol Song

Calden Wloka

Cem Yuksel

Ceyhun Akgul

Chang-Hun Kim

Changqing Zou

Changwen Zheng

Chaohui Wang

Chen Cao

Chen Zhao

Cheng Peng

Chenglei Wu

Cheng-Yuan Tang

Chetan Arora

Chi Kit Au

Chia-Chen Lin

Chirstian Duriez

Choi Ho-Hyoung

Christian Dick

Christof Rezk Salama

Chunlin Wu

Chunxia Xiao

Chunxiao Liu
Claudio Jung

Conglei Shi

Costas Sideris

Cuicui Kang

Daniel Pak-Kong Lun

Daniel Thalmann

Daniela Giorgi

David Chen

David Mould

Debaleena Chattopadhyay

Dhananjay Kumar

Di Huang

Dipl.-Math. Rasmus Buchmann

Donatello Conte

Dong-Ming Yan

Edmond Ho

Elias Wegert

Eric Haines

Erkang Cheng

Esdras Medeiros

Etienne Vouga

Eugene Zhang

Fabio Ganovelli

Fan Wang

Fan Zhong

Fang-Lue Zhang

Faouzi Ghorbel

Feng Lin

Filip Sadlo

Florian Baumann

Florian Nouviale

Francesco Banterle

Francisco Barranco

Francisco Feito

Franz - Erich Wolter

Frederic Cordier

Gael Guennebaud

Gael Sourimant

George Baciu

Georges-Pierre Bonneau

Gholamreza Anbarjafari

Glen Berseth

Guiqing Li

guoping wang

Guorong Li

Guoying Zhao

Haeyong Chung Chung

Hai Chao Zhu

Haibin Ling

Haiting Lin
Hamid Laga

Harald Reiterer

hareesh PV

Hassan Ugail

Hasup Lee

Heinrich Mueller

Helin Dutagaci

Hongbo Fu

Hongbo Zhou

Hongsong Li

Hongwei Lin

Hongzhi Wu

Hou Junhui

Hsueh-Yi Lin

Huang Hanming

Hui Ji

Hui Liang

Hung-Kuo Chu

Hwanyong Lee

Hyun Joon Shin

Igor Pandzic

Ikuhisa Mitsugami

Ingrid Hotz

In-Kwon Lee

Ioannis Marras

Ivan Viola

Ivana Kolingerova

Jacek Wolkowicz

Jean-Marie Normand

Jean-Philippe Vandeborre

Jia Li

Jia Pan

Jia-Bin Huang

Jianbing Shen

Jianchao Yang

Jianfeng Ren

Jiang Wang

Jianmin Li

Jianmin Zhang

Jianping Shi

Jihad El-Sana

Jin Huang

Jing Liao

Jinman Kim

Jinwei Gu

Jinwei Ye

Jiri Kosinka

João Paulo Lima

Jonas Mureika

Ju Hong Yoon 
Juan wachs

Jun $\mathrm{Wu}$

Jungho Yoon

Junping Zhang

Junqiu Wang

Junyong Noh

Juraj Vanek

Kai Berger

Kai Xu

Karoly Zsolnai

Katja Bühler

Kazufumi Kaneda

Kin Chung Kwan

Konstantinos Derpanis

krivanek Jaroslav

Kun Xu

Laszlo Neumann

Laszlo Szirmay-Kalos

Lee Jun

Lei He

Lei Li

Lei Wang

Lei Zhang

Leonardo De-Maeztu

Li Xu

Li-an Tang

Liangliang Nan

Lifeng Zhu

Ligang Liu

Lihua You

Liming Chen

Linh $\mathrm{Ha}$

Loic Barthe

Lorena Petrusca

Ludovic Hoyet

Luis Lopez

M. Brandon Haworth

Manfred Lau

Marc Nienhaus

Marcel Lancelle

Marcelo Kallmann

Marcelo Walter

Marco Agus

Marco Attene

Marco Muselli

Margarita Favorskaya

Markus Hadwiger

Markus Ihmsen

Martin Constable

Martin prazak
Mathias Paulin

Mathieu Bredif

Matthias Hullin

Maximilian Klein

Michael Weinmann

Min Tang

Minas Spetsakis

Mingliang Xu

Ming-Ming Cheng

Miquel Feixas Feixas

Mohammad Irfan

Mubbasir Kapadia

Mukta Prasad

Nakhoon Baek

Minas Spetsakis

Mingliang Xu

Ming-Ming Cheng

Miquel Feixas Feixas

Mohammad Irfan

Mubbasir Kapadia

Mukta Prasad

Nakhoon Baek

Neeharika Adabala

Nelson Chu

Ngai Ming Kwok

Nianjuan Jiang

Nic Marquardt

Nicolas Bonneel

Nicolas Pronost

Niels Christensen

Nivan Ferreira

Oliver Mattausch

Oliver van Kaick

Olivier Le Meur

Oswald Lanz

Panayiotis Charalambous

Paolo Bottoni

Pascal Barla

Pascal Berard

Patric Ljung

Patrick Callet

Paul Rosin

Paul Siebert

Peng Du

Peter Hall

Peter Rautek

Pierre Boulanger

Pierre Poulin

Pierre-Yves Laffont

Piyu Tsai
Prem Kalra

Przemyslaw Musialski

Przemyslaw Rokita

Qiang Cheng

Qiming Hou

Quentin AVRIL

Radu Timofte

Ralph Martin

Ramakrishna Kakarala

Ramsay Dyer

Ran Margolin

Raoul Wessel

Remco Veltkamp

Ren Mao

Renjie Chen

Rocco Gasteiger

Romain Vergne

Roman VLASOV

Ronald Peikert

Ronan Boulic

Rubens Nunes

Rüdiger Westermann

Rui Wang

Ruofeng Tong

Ryoichi Ando

Sagi Schein

Sagi Schein

Samuel Lemercier

Sang Il Park

Sang Ok Koo

Scott Schaefer

Selcuk Sumengen

Selim Balcisoy

Shang-Hong Lai

Shanmuganathan Raman

Shanmuganathan Raman

Shaoting Zhang

Shiguang Shan

Shi-Min $\mathrm{Hu}$

Shiqing Xin

Shizhe Zhou

Shuai Fang

Silvia Biasotti

Sofien Bouaziz

Song-Hai Zhang

Song-Pei Du

Soo Min Kang

Soong-Der Chen

Soraia Musse

Stefano Berretti 
Stephane Nicolau

Steve Maddock

Subodh Kumar

Sumant Pattanaik

Sung Hee Lee

Sungkil Lee

Susanne Suter

Svetlana Stolpner

Tae-Joon Kim

Taesoo Kwon

Tam Nguyen

Tao Chen

Thomas Auzinger

Thomas Wischgoll

Tian Lan

Tianjia Shao

Tianlu Mao

Tianzhu Zhang

Tolga Capin

Tsz Ho Kwok

Ulrich Rückert

Vedad Hulusic

Vladimir Kim

W Randolph Franklin

Wanqing Li

Wei Chen

Wei Shen

Weidong Geng

Weiming Dong

Weiwei Cui

Wenzel Jacob

Wien Hong

William, Lai Chi-Fu

Wu Jing
Xianbiao Qi

Xianfang Sun

Xiao Yang

Xiaobai Chen

Xiaogang Jin

Xiaohu Guo

Xiaolei Huang

Xiaopeng Hong

Xiaoru Yuan

Xiaotian Yin

Xiaoyang Tan

Xin Li

Xin Sun

Xinbo Gao

Xinyu Zhang

Xioayang Mao

Xuan Song

Xudong JIANG

Xudong Kang

Xue Dong Yang

Xuejin Chen

Xuemei Zhao

Xueting Liu

Yangang Wang

Yanwen Guo

Yasushi Makihara

Ye Luo

Yezhou Yang

Yi Gao

Yi Li

Yi Wu

Yi Wu

Yifei Lou

Yiying Tong
Yong Xia

Yonghuai Liu

Yong-Jin Liu

Yong-Liang Yang

Yongtao Lu

Yongwei Miao

Younhyun Jung

Youyi Zheng

Yu Ji

Yuanyuan Ding

Yuchao Dai

Yu-Chi Lai

Yu-Kun Lai

Yun Sheng

Yun Zhang

Yung-Yu Chuang

Yu-Shuen Wang

Yusuf Sinan Akgül

Yves Lucas

Zeng-Fu Wang

Zhanghui Kuang

Zhaoqi Wang

Zhe Hu

Zheng Changxi

Zhenpeng Bian

Zhi Liu

Zhichun Mu

Zhong Ren

Zhonggui Chen

Zhouhui Lian

Zhuming Zhang

Ziyang Ma 